detected sequences have a biological function. Here, the main question is whether or not TEs within or in the vicinity of asthma susceptible genes modulate the gene expression of these genes. This can be performed using cap-analysis gene expression, a method that specifically and unequivocally identifies the exact transcription start sites of genes [6]. Once the regulated retrotransposon transcriptome of healthy tissue is known, it can be compared with that of asthma patients or additional complex diseases. This will provide unprecedented details about genes and gene expression patterns, and how they relate to the development and/or establishment of complex diseases. The impact of TEs on phenotype (variation) and disease are depicted in figure 1.

With the advent of next generation sequencing and rapid genome and transcriptome analyses, the dynamic nature of TEs and their role in shaping intra- and inter-individual variation may soon be resolved. Because these developments will dissect TE-induced variation at ever increasing resolution, they will lead to a better understanding of TEs and their overall impact on gene expression, human health and the (epi)genetic constitution that underlies complex lung diseases, including asthma. We have already identified the number, position and type of TEs present in the 12 aforementioned asthma-associated genes and we are in the process of screening the same panel of genes in asthma patients. It may well be that some phenotypes of asthma are predominantly associated with TE-regulated genes of the immunological compartment, whereas other phenotypes of asthma are linked to a specific set of TE-regulated genes in lung tissue cells. Therefore, cell- and phenotype-specific understanding of the healthy and diseased transcriptomes may lead to better treatment and to personalised medicine. We would like to encourage pulmonary scientists to further examine the almost entirely unexplored TE field, which integrates clinics, the laboratory and bioinformatics. The development of entire novel strategies to treat complex lung diseases should be envisaged.

Nicola Miglino*, Michael Roth*, Florent Baty", Martin Brutsche ${ }^{\#}$, Michael Tamm* and Pieter Borger*

*Pulmonary Cell Research, Dept of Biomedicine, University Hospital Basel, Basel, and "Dept of Pneumology, Kantonsspital St Gallen, St Gallen, Switzerland.
Correspondence: P. Borger, Pulmonary Cell Research, Dept of Biomedicine, University of Basel, Hebelstrasse 20, CH-4031 Basel, Switzerland. E-mail: pieter.borger@unibas.ch

Support Statement: M. Roth, M. Tamm and P. Borger received research grants from the Swiss National Science Foundation.

Statement of Interest: None declared.

\section{REFERENCES}

1 Levy S, Sutton G, $\mathrm{Ng}$ PC, et al. The diploid genome sequence of an individual human. PLoS Biol 2007; 5: e254.

2 Cordaux R, Batzer MA. The impact of retrotransposons on human genome evolution. Nat Rev Genet 2009; 10: 691-703.

3 Faulkner GJ, Carninci P. Altruisctic functions of selfish DNA. Cell Cycle 2009; 15: 2895-2900.

4 Bannert N, Kurth R. Retroelements and the human genome: new perspectives on an old relation. Proc Natl Acad Sci USA 2004; 101: Suppl. 2, 14572-14579.

5 Sela N, Mersch B, Gal-Mark N, et al. Comparative analysis of transposed element insertion within human and mouse genomes reveals Alu's unique role in shaping the human transcriptome. Genome Biol 2007; 8: R127.

6 Faulkner GJ, Kimura Y, Daub CO, et al. The regulated retrotransposon transcriptome of mammalian cells. Nat Genet 2009; 41: 563-571.

7 O'Donnel KA, Burns KH. Mobilizing diversity: transposable element insertions in genetic variation and disease. Mob DNA 2010; 1: 21.

8 Bush A, Menzies-Gow A. Phenotypic differences between pediatric and adult asthma. Proc Am Thorac Soc 2009; 6: 712-719.

9 Postma DS. Gender differences in asthma development and progression. Gend Med 2007; 4: Suppl. B, S133-S146.

10 Postma DS, Koppelman G. Genetics of asthma: where are we and where do we go? Proc Am Thorac Soc 2009; 6: 283-287.

11 E Paraskakis, G Sourvinos, Passam F, et al. Microsatellite DNA instability and loss of heterozygosity in bronchial asthma. Eur Respir J 2003; 22: 951-955.

12 Lialiaris T, Polyzou A, Mpountoukas P, et al. Chromosome instability on children with asthma. J Asthma 2009; 46: 841-844.

13 Cockerill PN, Shannon MF, Bert AG, et al. The Granulocytemacrophage colony-stimulating factor/interleukin-3 locus is regulated by an inducible cyclosporine A sensitive enhancer. Proc Natl Acad Sci USA 1993; 90: 2466-2470.

DOI: $10.1183 / 09031936.00189811$

\title{
Negative predictive value of TST and IGRA in anti-TNF treated patients
}

\section{To the Editors:}

Anti-tumour necrosis factor (TNF) therapy is increasingly used in chronic inflammatory diseases. These agents are related to an increased risk of tuberculosis (TB) depending on the clinical setting and the anti-TNF agent used, so screening is mandatory before its start [1]. Inhibition of TNF- $\alpha$ and TNF-regulated chemokine networks is the presumed biological basis [2]. Though patients with chronic inflammatory diseases and suspected latent TB infection (LTBI) who are candidates for anti-TNF therapy are at higher risk of TB reactivation, they are prone to false-negative tuberculin skin test (TST) and interferon- $\gamma$ release assay (IGRA) results due to T-cell hyporesponsiveness, either because of illness or immunosuppressive medications [3]. The reliability of these tests in this population is therefore of interest, but currently unclear. The negative predictive value (NPV) of TST and IGRA is 
still not defined in this group of patients. National guidelines for diagnosis and treatment of LTBI require screening of any candidate on anti-TNF therapy, prior to starting it, through assessment of TB signs and symptoms, chest radiography, TST and IGRA. After exclusion of active TB, a positive TST $(\geqslant 10 \mathrm{~mm}$ in immunocompetent or $\geqslant 5 \mathrm{~mm}$ in immunocompromised patients) and/or positive IGRA, radiologic evidence of previously untreated TB or recent contact with TB guarantee preventive therapy [4]. Our aim was to determine the NPV of TST and IGRA for progression to disease in patients on anti-TNF therapy who did not start preventive therapy. We conducted a retrospective analysis of all anti-TNF candidates screened for TB (January 2008 to June 2010) at the Chest Diagnosis Centre of Vila Nova de Gaia outpatient TB clinic (Vila Nova de Gaia, Portugal), according to previously described national guidelines. Follow-up was maintained until January 2012, with careful evaluation of new symptoms suggestive of active disease while patients received anti-TNF therapy. Demographic and clinical data were collected from clinical registries. The NPV for TST and IGRA in patients who were not started on preventive therapy and were treated with antiTNF therapy was calculated. The IGRA method used was QuantiFERON®-TB Gold in-Tube (Cellestis, Carnegie, Australia).

During the studied period 168 patients were screened for TB, in order to start biological treatment. 10 of them were lost during follow-up, both in the TB unit and the medical clinics that first sent them and never started biological treatment. In our evaluation we included 158 patients, 81 (51.3\%) males and 77 $(48.7 \%)$ females, with mean \pm SD age of $42.8 \pm 12.6$ yrs. $85(53.8 \%)$ patients were on immunosuppressive drugs when screened, most frequently steroid ( $\geqslant 15 \mathrm{mg} \mathrm{day}^{-1}$ prednisolone or equivalent) therapy $(27.1 \%)$, or azathioprine and metothrexate (both $25.9 \%$ ) alone or in combination with steroids. 80 (9.4\%) patients were already on anti-TNF therapy with natalizumab. Of the 158 patients, $86(54.4 \%)$ had positive screening; after exclusion of active TB they were started on preventive therapy and $86.0 \%$ of them successfully completed (9 months of isoniazid or 4 months of rifampin because of toxicity or intolerance to isoniazid). 58 patients who screened positive started anti-TNF therapy 4 weeks after the start of preventive therapy. There were no reported TB cases in the positive screening group. Of the $72(45.6 \%)$ patients screened negative, $27(37.5 \%)$ were on immunosuppressive therapy at the time of screening; of these, $24(88.9 \%)$ were started on anti-TNF therapy, one of whom developed active TB. The remaining $45(62.5 \%)$ patients who screened negative were not on immunosuppressive drugs; of these, 35 (77.8\%) were started on anti-TNF therapy, and there were no reported TB cases. Taking into account the results obtained, a NPV for progression to disease (for TST and IGRA) was $95.8 \%$ (95\% CI 0.769-0.998) in the immunocompromised and $100 \%$ (95\% CI 0.876-1.000) in immunocompetent patients.

Since all patients who did not receive preventive therapy had, by definition, normal radiologic studies (chest radiography or tomography whenever chest radiography raised doubts) and no history of previous TB or TB contacts, the NPV for TST and IGRA could safely be defined. Despite thorough investigation, one immunocompromised patient developed active disease; a 61-yrold female with rheumatoid arthritis, on $25 \mathrm{mg}$ oral prednisolone at the time of investigation. She screened negative for both TST and IGRA and had no history of contacts with TB, but developed active TB 22 months after initiation of adalimumab, reminding us that false negative results may still occur, particularly if the patient is already taking immunossupressive therapy. BELARD et al. [5] observed in his study that oral prednisolone severely suppressed IGRA and TST performance, recommending screening prior to initiation of prednisolone therapy.

It is important to stress, however, that in our patient the long interval between initiation of anti-TNF agent and development of disease could be due to recent infection, and not reactivation of LTBI.

The main limitations of our study were its retrospective design and the reduced sample of patients who were actually started on anti-TNF agents.

Our study suggests that the NPV of available TB screening tests may be higher in immunocompetent patients, so TB screening should be performed at an early stage of disease and before starting any immunosuppressive therapy. These results warrant confirmatory studies in a larger population.

\section{Sergio Campainha*, Teresa Gomes ${ }^{\#}$, Aurora Carvalho* ${ }^{*}$ and Raquel Duarte ${ }^{*},++$ \\ *Pulmonology Dept, Vila Nova de Gaia/Espinho Hospital Centre, 'Chest Diagnosis Centre of Vila Nova de Gaia, Vila Nova de Gaia, "Pulmonology Dept, Tras-os-Montes e Alto Douro Hospital Centre, Vila Real, and ${ }^{+}$Dept of Clinical Epidemiology, Predictive Medicine and Public Health University of Porto Medical School, Porto, Portugal.}

Correspondence: S. Campainha, Servico de Pneumologia, Centro Hospitalar de Vila Nova de Gaia, Rua Conceicao Fernandes s/n, 4434/502 Vila Nova de Gaia, Portugal. E-mail: sergio.fernandes@chvng.min-saude.pt

Statement of Interest: None declared.

\section{REFERENCES}

1 Solovic I, Sester M, Gomez-Reino R, et al. The risk of tuberculosis related to tumour necrosis factor antagonist therapies: a TBNET consensus statement. Eur Respir J 2010; 36: 1185-1206.

2 Algood MH, Lin PL, Flynn JL. Tumor necrosis factor and chemokine interactions in the formation and maintenance of granulomas in tuberculosis. Clin Infect Dis 2005; 41: Suppl. 3, S189-S193.

3 Keystone EC, Papp KA, Wobeser W. Challenges in diagnosing latent tuberculosis infection in patients treated with tumor necrosis factor antagonists. J Rheumatol 2011; 38: 1234-1243.

4 Fonseca J, Lucas H, Canhão H, et al. Guidelines for the diagnosis and treatment of latent tuberculosis infection and active tuberculosis in patients with inflammatory joint diseases proposed for treatment with tumor necrosis factor alpha antagonists drugs. Rev Port Pneumol 2006; 12: 603-613.

5 Bélard E, Semb S, Ruhwald M, et al. Prednisolone treatment affects the performance of the QuantiFERON gold in-tube test and the tuberculin skin test in patients with autoimmune disorders screened for latent tuberculosis infection. Inflamm Bowel Dis 2011; 17: $2340-2349$.

DOI: $10.1183 / 09031936.00161711$ 